\title{
Albuminuria Is Associated with Steatosis Burden in Patients with Type 2 Diabetes Mellitus and Nonalcoholic Fatty Liver Disease (Diabetes Metab J 2021;45:698-707)
}

\author{
Eugene Han, Hye Soon Kim \\ Department of Internal Medicine, Keimyung University School of Medicine, Daegu, Korea
}

We are grateful for being provided the opportunity to respond to the letter received from Prof. Mi-kyung Kim concerning our recent article in Diabetes and Metabolism Journal entitled "Albuminuria is associated with steatosis burden in patients with type 2 diabetes mellitus and nonalcoholic fatty liver disease." Further, we would like to extend our gratitude to Prof. Mikyung Kim for her insightful comments on our study. In this study, patients with type 2 diabetes mellitus and nonalcoholic fatty liver disease (NAFLD) were enrolled using prospective method, and the data was collected in a cross-sectional design. Given that the Korean health insurance does not cover elastography, its high cost would be an economic burden for some patients. Owing to this, data from only a few patients that had undergone elastography was collected in a retrospective manner. Furthermore, the grant covered the examination fee for the patients included in the prospective design [1]. We would also like to thank the Korean Diabetes Association for supporting this study. We believe that the outcomes of our study outline the clinical implication of albuminuria and signify the importance of identifying high risk NAFLD patients via elastography. In particular, the recent expert consensus guidelines in both America and Europe emphasize the need for evaluating the degree of hepatic steatosis in NAFLD patients with metabolic risk factors [2,3]. With respect to the impact of metformin on NAFLD, a cut-off value of $45 \mathrm{~mL} / \mathrm{min} / 1.73 \mathrm{~m}^{2}$, which is the borderline for metformin treatment, was applied [4]. In addition, there are comparable outcomes of chronic kidney disease
(CKD) stage II and CKD stage III A $[5,6]$. As per Prof. Mikyung Kim's suggestion, we categorized the study population based on stages of liver stiffness. A few patients with advanced liver fibrosis (liver stiffness measurement $\geq 9.3 \mathrm{kPa}, n=12$ ) exhibited insignificant differences in albuminuria compared to those without advanced liver fibrosis. Our study population included relatively younger subjects with shorter course of diabetes. Therefore, it is possible that patients with earlier stages of NAFLD compared with that of a previous Chinese study [7] may have been enrolled, and thus, distinct results were obtained.

We agree with Dr. Mi-kyung Kim that prospective, further longitudinal and well-designed trials are required to characterize albuminuria as a convincing marker for the progression of NAFLD in diabetes. As indicated by Prof. Mi-kyung Kim, we believe that albuminuria could play a major role in identifying the link between NAFLD and diabetes.

\section{CONFLICTS OF INTEREST}

No potential conflict of interest relevant to this article was reported.

\section{REFERENCES}

1. Han E, Kim MK, Jang BK, Kim HS. Albuminuria is associated with steatosis burden in patients with type 2 diabetes mellitus
Corresponding author: Hye Soon Kim (D) https://orcid.org/0000-0001-6298-3506 Department of Internal Medicine, Keimyung University School of Medicine, 1095 Dalgubeol-daero, Dalseo-gu, Daegu 42601, Korea

E-mail: hsk12@dsmc.or.kr
This is an Open Access article distributed under the terms of the Creative Commons Attribution Non-Commercial License (https://creativecommons.org/licenses/by-nc/4.0/) which permits unrestricted non-commercial use, distribution, and reproduction in any medium, provided the original work is properly cited. 
and nonalcoholic fatty liver disease. Diabetes Metab J 2021;45: 698-707.

2. European Association for the Study of the Liver; Clinical Practice Guideline Panel; EASL Governing Board representative. EASL clinical practice guidelines on non-invasive tests for evaluation of liver disease severity and prognosis: 2021 update. J Hepatol 2021;75:659-89.

3. Ando Y, Jou JH. Nonalcoholic fatty liver disease and recent guideline updates. Clin Liver Dis (Hoboken) 2021;17:23-8.

4. Hur KY, Kim MK, Ko SH, Han M, Lee DW, Kwon HS, et al. Metformin treatment for patients with diabetes and chronic kidney disease: a Korean Diabetes Association and Korean So- ciety of Nephrology Consensus Statement. Diabetes Metab J 2020;44:3-10.

5. Liu C, Chen H, Liu C, Fu C, Zhang H, Yang H, et al. Combined application of eGFR and albuminuria for the precise diagnosis of stage 2 and 3a CKD in the elderly. J Nephrol 2014;27:289-97.

6. Shardlow A, McIntyre NJ, Fluck RJ, McIntyre CW, Taal MW. Chronic kidney disease in primary care: outcomes after five years in a prospective cohort study. PLoS Med 2016;13:e1002128.

7. Yeung MW, Wong GL, Choi KC, Luk AO, Kwok R, Shu SS, et al. Advanced liver fibrosis but not steatosis is independently associated with albuminuria in Chinese patients with type 2 diabetes. J Hepatol 2018;68:147-56. 